\title{
Exact knot solutions in a generalized Skyrme-Faddeev model
}

\author{
L. P. Zou, ${ }^{1,2,3}$ P. M. Zhang, ${ }^{1,4}$ and D. G. Pak ${ }^{1,5}$ \\ ${ }^{1}$ Institute of Modern Physics, Chinese Academy of Sciences, Lanzhou 730000, China \\ ${ }^{2}$ School of Nuclear Science and Technology, Lanzhou University, Lanzhou 730000, China \\ 3 University of Chinese Academy of Sciences, Beijing 100049, China \\ ${ }^{4}$ Research Center for Hadron and CSR Physics, Lanzhou University, Lanzhou 730000, China \\ ${ }^{5}$ Lab. of Few Nucleon Systems, Institute for Nuclear Physics, Ulugbek, 100214, Uzbekistan
}

\begin{abstract}
We propose a generalized Skyrme-Faddeev type theory with an additional scalar field. In a special case of model parameters one has a theory which admits exact knot solutions given by a class of exact toroidal solitons from Aratyn-Ferreira-Zimerman (AFZ) integrable $C P^{1}$ model. In a general case the theory admits an exact knot solution for a unit Hopf charge. For higher Hopf charges we perform numeric analysis of the solutions and obtain estimates for the knot energies using energy minimization procedure based on ansatz with AFZ field configurations and with rational functions. We show that AFZ configurations provide a better approximate solutions. The corresponding knot energies are in a good agreement with a standard law for the low energy bound, $E_{H} \simeq Q_{H}^{3 / 4}$.
\end{abstract}

PACS numbers: 11.15.-q, 14.20.Dh, 12.38.-t, 12.20.-m

Keywords: Knot solitons, Skyrme-Faddeev theory, effective QCD

Since invention of the original Skyrme theory [1, 2] a great development towards its generalizations and applications in description of baryons has been made [3] Topological solitons in that theory, so called Skyrmions, have been constructed numerically for a wide range of baryon numbers [6, 7]. The Skyrme-Faddeev theory [8, 9] represents another kind of effective theories which supposed to be induced from quantum chromodynamics (QCD) in low energy regime. The theory possesses topological knot solitons classified by the homotopy group $\pi_{3}\left(C P^{1}\right)=Z$, i.e., by the topological Hopf charge. It is proposed to interpret such knot solutions as color electric and color magnetic glueball states [8, 10 13. One should notice, that derivation of a strict expression for a low energy effective action from the basics of QCD is an extremely difficult problem [14 16]. So far there exists a number of various extended Skyrme-Faddeev models where some exact and numeric knot and vortex solutions have been found [17-20].

In the present paper we consider an extended SkyrmeFaddeev model with an additional scalar field. In general the low energy effective action of QCD contains scalar fields [10] which originate from unknown coefficient correlation functions in the effective action and play roles of order parameters in the effective theory 14. We consider a minimal extension of the Skyrme-Faddeev theory to find out a model which is similar to the Skyrme theory, and, at the same time, inherits properties of integrable $C P^{1}$ models.

So far, all known topological solutions in $(3+1)$ Skyrme type models are obtained numerically except the original Skyrmion soliton [1]. Some exact vortex solutions have been obtained in a generalized Skyrme-Faddeev theory for a special set of model parameters which imply the existence of integrable submodel in the theory [17-19]. A family of exact analytic knot solutions with toroidal configuration have been constructed in special integrable $C P^{1}$ models [21, 22]. Unfortunately, physical applica- tions of such solutions in QCD remain unclear. We propose a Skyrme-Faddeev-type model which admits exact knot solutions and which can give some hints towards constructing a more realistic low energy QCD effective theory.

Let us consider an extended Skyrme-Faddeev model defined by the following Lagrangian

$$
\begin{aligned}
\mathcal{L}(\phi, \hat{n})= & -\mu^{2}\left(\partial_{\mu} \phi\right)^{2}-\frac{\beta}{4} \phi^{2}\left(\partial_{\mu} \hat{n}\right)^{2}-\frac{\nu}{32} \frac{1}{\phi^{2}} H_{\mu \nu}^{2}- \\
& \frac{\xi}{32} \frac{1}{\phi^{2}}\left(\partial_{\mu} \hat{n}\right)^{4},
\end{aligned}
$$

where $\hat{n}$ is a unit triplet field in adjoint representation of $S U(2)$ color group, i.e., $C P^{1}$ field, $\phi$ is a real scalar field, and $H_{\mu \nu}$ is a magnetic field defined as follows

$$
H_{\mu \nu}=\epsilon^{a b c} \hat{n}^{a} \partial_{\mu} \hat{n}^{b} \partial_{\nu} \hat{n}^{c},
$$

$\mu, \beta, \nu, \xi$ are model parameters. The first two terms in (1) coincide with respective first two terms in the original Skyrme Lagrangian after proper changing variable for the scalar field. The last term in the Lagrangian appears in the one-loop effective action of standard QCD as it was shown in 14, 16. The topological content of the theory is determined by the $C P^{1}$ field $\hat{n}$ realizing the Hopf mapping. One has two non-trivial homotopy groups, $\pi_{2}\left(C P^{1}\right)=Z$ and $\pi_{3}\left(C P^{1}\right)=Z$, which classify all non-equivalent topological classes of $\hat{n}$ by the monopole and Hopf charges respectively. Like Skyrme theory, the model defined by the Lagrangian (1) reduces to the Skyrme-Faddeev theory in the limit of constant scalar field and $\xi=0$. So that, our model admits knot solutions and a singular Wu-Yang monopole solution given by the hedgehog configuration $\hat{n}=\frac{\vec{r}}{r}$.

Using stereographic projection it is convenient to introduce a complex function $u(x)$ which parameterizes the two dimensional sphere $S^{2} \simeq C P^{1}$. As usually, we assume that three-dimensional space $R^{3}$ is compactified to 
a three-sphere $S^{3}$ by imposing appropriate boundary conditions at space infinity. With this one has the following expression for the field $\hat{n}$ in terms of the complex function $u(x)$

$$
\hat{n}=\frac{1}{1+u \stackrel{*}{u}}\left(\begin{array}{c}
u+\stackrel{*}{u} \\
-i(u-\stackrel{*}{u}) \\
u \stackrel{*}{u}-1
\end{array}\right) .
$$

The magnetic field $H_{\mu \nu}$ can be written explicitly in terms of $u$ as well

$$
H_{\mu \nu}=\frac{-2 i}{\left(1+|u|^{2}\right)^{2}}\left(\partial_{\mu} u \partial_{\nu} \stackrel{*}{u}-\partial_{\nu} u \partial_{\mu} \stackrel{*}{u}\right) .
$$

Since the magnetic field $H_{\mu \nu}$ represents a closed differential two-form, one can find a corresponding dual Abelian magnetic potential $\tilde{C}_{\mu}$

$$
H_{\mu \nu}=\partial_{\mu} \tilde{C}_{\nu}-\partial_{\nu} \tilde{C}_{\mu} .
$$

The Hopf and monopole charges for a given mapping $u(x)$ can be calculated as follows $(i, j=1,2,3)$

$$
\begin{aligned}
Q_{H} & =\frac{1}{32 \pi^{2}} \int d^{3} x \epsilon^{i j k} \tilde{C}_{i} H_{j k}, \\
g_{m} & =\int_{S^{2}} H_{i j} d \sigma^{i j} .
\end{aligned}
$$

Let us remind the main result obtained in study of Aratyn-Ferreira-Zimerman integrable model 22. The Lagrangian of AFZ model is given by

$$
\mathcal{L}_{A F Z}=-\eta_{0}\left(H_{\mu \nu}^{2}\right)^{\frac{3}{4}} .
$$

The model possesses a family of exact knot solutions with a Hopf charge $Q_{H}=m n$ expressed in terms of two winding numbers $(m, n)$. For our further purpose it is suitable to rewrite the analytic solutions of AFZ model in spherical coordinates. In the case of equalled winding numbers, $m=n$, the solution reads

$$
\begin{aligned}
u= & \frac{e^{i m \phi}}{2 a r \sin \theta}\left(2 a r \cos \theta-i\left(a^{2}-r^{2}\right)\right) \\
& \left(\frac{2 a r \cos \theta-i\left(a^{2}-r^{2}\right)}{\sqrt{\left(a^{2}+r^{2}\right)^{2}-4 a^{2} r^{2} \sin ^{2} \theta}}\right)^{m-1},
\end{aligned}
$$

where $a$ is a free parameter characterizing the size of the knot. In the case of $m \neq n$ an exact knot solution takes the form

$$
\begin{aligned}
& u= e^{i n \phi}\left(\frac{2 a r \cos \theta-i\left(a^{2}-r^{2}\right)}{d}\right)^{m} . \\
&\left(-\frac{a^{2}+r^{2}-\sqrt{\frac{n^{2} d}{m^{2}}+4 a^{2} r^{2} \sin ^{2} \theta}}{a^{2}+r^{2}-\sqrt{d+\frac{4 m^{2} a^{2} r^{2} \sin ^{2} \theta}{n^{2}}}}\right)^{\frac{1}{2}}, \\
& d \equiv a^{4}+r^{4}+2 a^{2} r^{2} \cos (2 \theta) .
\end{aligned}
$$

Let us consider first a special case of a reduced Lagrangian obtained from (1) by setting a condition $\beta=$ 0 . In this case one can find exact knot solutions with Hopf charges determined by equalled winding numbers, $Q_{H}=m^{2}$. By explicit calculating we have checked for Hopf charges $Q_{H}$ corresponding to winding numbers $m=n=1,2, \ldots, 6$ that field configurations 8 and the scalar field given by

$$
\phi=\frac{c_{0}}{\sqrt{a^{2}+r^{2}}},
$$

provide exact solutions to all equations of motion under the following constraint

$$
\mu^{2}-\frac{8 m^{2}(\nu+4 \xi)}{3 a^{2}}=0 .
$$

The integration constant $c_{0}$ in 10 is fixed by boundary condition for $\phi$ at the origin. In analogy with the Skyrme theory we will impose the following boundary conditions for the scalar field

$$
\phi(r=0)=1, \quad \phi(r=\infty)=0 .
$$

This implies $c_{0}=a$. The constraint (11) allows to determine the knot size parameter

$$
a_{m}^{2}=\frac{8 m^{2}(\nu+4 \xi)}{3 \mu^{2}} .
$$

A corresponding energy density of the solution is

$$
\mathcal{E}_{m}=\frac{8 m^{2}(\nu+4 \xi)\left(r^{2}+3 a_{m}^{2}\right)}{3\left(a_{m}^{2}+r^{2}\right)^{3}} .
$$

Integrating the energy density over space results in the following expression for the total energy of the soliton

$$
E_{m}=4 \pi^{2} \mu Q_{H}^{\frac{1}{2}} \sqrt{\frac{3}{8}(\nu+4 \xi)} .
$$

Notice, the energy of the knot is proportional to $Q_{H}^{\frac{1}{2}}$, this is contrary to the usually expected dependence of the low energy bound on the Hopf charge, $E_{\text {bound }} \simeq Q_{H}^{3 / 4}$, found in a non-linear sigma model [23]. It would be interesting to find exact solutions for the Hopf charge given by non-equalled winding numbers, $m \neq n$, to verify whether the energy of knot solitons in that case will have a total energy expressed by the same equation (15).

Let us consider a general case when the parameter $\beta$ does not vanish. By direct calculating all equations of motion one can check that an exact analytic solution with a unit Hopf charge, $Q_{H}=1$, is given by the same expressions 10. 8) for $m=n=1$ and when the following condition is fulfilled

$$
\frac{3}{8} \mu^{2}+\beta-\frac{\nu+4 \xi}{a^{2}}=0
$$


If we keep the model parameters $\mu, \beta, \nu, \xi$ arbitrary the condition will fix the value of the knot size

$$
a_{1}^{2}=\frac{\nu+4 \xi}{\frac{3}{8} \mu^{2}+\beta} .
$$

It is easy to write down explicit expressions for the components of the magnetic field

$$
\begin{aligned}
H_{r \theta} & =-\frac{32 r^{2} \sin \theta}{\left(a^{2}+r^{2}\right)^{3}}, \\
H_{\theta \phi} & =-\frac{8 r^{2} \sin (2 \theta)}{\left(a^{2}+r^{2}\right)^{2}}, \\
H_{r \phi} & =-\frac{8 r\left(a^{2}-r^{2}\right)(1-\cos (2 \theta)}{\left(a^{2}+r^{2}\right)^{3}} .
\end{aligned}
$$

Vector field lines of the magnetic field projected onto the planes $X O Z$ and $X O Y$ are depicted in Figs. 1,2 (in cartesian coordinates $x, y, z)$. One can verify that magnetic fluxes through the circle of radius "a" in the plane $z=0$ and through the half plane $y>0$ are quantized with a factor $4 \pi$ which is twice larger than a corresponding factor of magnetic flux quantization in the Maxwell theory

$$
\begin{gathered}
\Phi_{m}=\int H_{r \phi} d r d \phi=4 \pi m, \\
\Phi_{n}=\int H_{r \theta} d r d \theta=4 \pi n .
\end{gathered}
$$

So, the Hopf charge $Q_{H}=m n$ provides the linking number for the magnetic field which has a helical toroidal structure.

One can calculate the energy density for the knot with a unit Hopf charge

$$
\mathcal{E}_{1}=\frac{a_{1}^{2}\left(8\left(\beta a_{1}^{2}+4 \xi+\nu\right)+\mu^{2} r^{2}\right)}{\left(a_{1}^{2}+r^{2}\right)^{3}} .
$$

This implies the total energy

$$
E_{1}=4 \pi^{2} \sqrt{\left(\beta+\frac{3}{8} \mu^{2}\right)(\nu+4 \xi)} .
$$

Surprisingly, as it was observed first in 21], the knot configuration has a very nice feature. Even though the magnetic field possesses a toroidal topology, the energy density is completely spherically symmetric (see Fig. 2a).

For higher Hopf charges, $Q_{H}>1$, numeric analysis implies that solution for the scalar field $\phi$ is not sphericallysymmetric. To find approximate knot solutions with higher Hopf numbers we apply minimization procedure for the energy functional. A key point is how to find a proper ansatz for the function $u(x)$ which could provide a minimum energy for a chosen Hopf charge. We apply first the ansatz for $u(x)$ given by AFZ configurations (9). To find a dominant contribution to the energy we introduce the following ansatz for the scalar field $\phi$

$$
\phi=\frac{c_{1}}{\sqrt{c_{2}^{2}+r^{2}}}+\frac{c_{3}}{\sqrt{c_{4}^{2}+r^{2}}} \sin \theta
$$

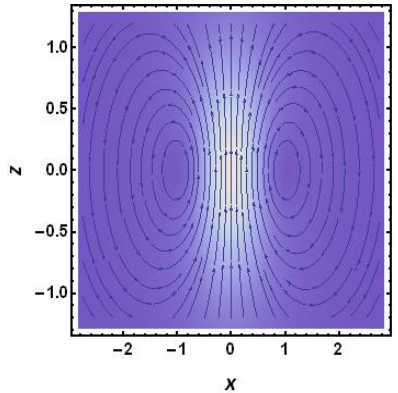

(a)

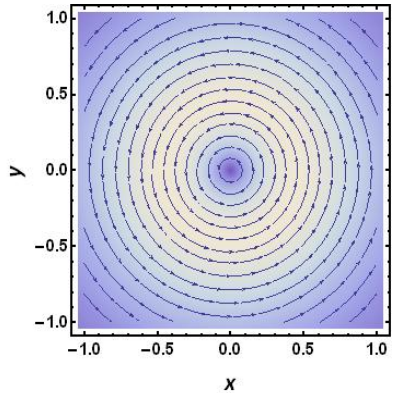

(b)
FIG. 1: Vector lines of the magnetic field projected onto the planes $y=0,(\mathrm{a})$, and $z=0,(\mathrm{~b})$.

where $c_{1}, c_{2}, c_{3}, c_{4}$ are variational number parameters. We set the values of the model parameters in the Lagrangian (1) to $(\xi=0, \beta=0.33, \mu=1, \nu=1.5)$. Without loss of generality we put $\xi=0$ since the presence of the last term in (1) does not change a qualitative picture of the solution structure. Minimization of the energy with the ansatz based on AFZ solutions leads to the following estimates for the total energy and knot size parameter $a$ given in Table I. One can see that dependence of the knot energy on Hopf charge is in a good agreement with the low energy bound found in 23 .

$$
E_{\text {bound }}=E_{1} Q_{H}^{3 / 4} \text {. }
$$

A significant discrepancy appears for knots with Hopf charge defined by equalled winding numbers, $Q_{H}=m^{2}$. For instance, for Hopf charge $Q_{H}=4, m=n=2$ the energy minimization procedure with the ansatz 8100 produces energy value $E_{4}(m=n=2)=105.7$ which is less than a respective low energy bound. This is caused by the essential feature of the AFZ configurations, namely, by the spherical symmetry of the energy densities for $m=n=1,2$ (see Figs. 2a, 2d).

Let us now consider the ansatz with rational functions $u\left(z_{1}, z_{2}\right)$ which was successfully applied in the SkyrmeFaddeev model 25]. The ansatz starts with the definition of mapping $R^{3} \rightarrow S U(2) \simeq S^{3}$

$$
\begin{aligned}
& z_{1}=\frac{x_{1}+i x_{2}}{r} \sin f(r), \\
& z_{2}=\cos f(r)+i \frac{x_{3}}{r} \sin f(r),
\end{aligned}
$$

where $f(r)$ is a trial function, and the complex coordinates $z_{1}, z_{2}$ describe the sphere $S^{3}$ imbedded into the complex plane $C^{2}$

$$
\left|z_{1}\right|^{2}+\left|z_{2}\right|^{2}=1
$$

A class of toroidal knot configurations of type $\mathcal{A}_{m, n}$ with a Hopf charge $Q_{H}=m n$ is defined by the rational function [25]

$$
u=\frac{z_{2}^{m}}{z_{1}^{n}}
$$




\begin{tabular}{|c|c|c|c|c|c|}
\hline$(\mathrm{m}, \mathrm{n})$ & $Q_{H}$ & $E_{\text {bound }}$ & $E_{A F Z}$ & $a_{A F Z}$ & $E_{\text {rat }}$ \\
\hline$(1,1)$ & 1 & 40.6 & 40.6 & 1.46 & 41.6 \\
\hline$(1,2)$ & 2 & 68.3 & 67.28 & 2.0 & 68.3 \\
\hline$(2,1)$ & 2 & 68.3 & 67.26 & 2.03 & 75.0 \\
\hline$(1,3)$ & 3 & 92.5 & 96.9 & 1.53 & 107.8 \\
\hline$(3,1)$ & 3 & 92.5 & 98.8 & 0.51 & 116 \\
\hline$(1,4)$ & 4 & 115 & 129 & 1.49 & 149 \\
\hline$(4,1)$ & 4 & 115 & 133 & 0.50 & 164 \\
\hline$(2,2)$ & 4 & 115 & 105.7 & 0.57 & 123 \\
\hline
\end{tabular}

TABLE I: Energy and knot size values for Hopf charge $Q_{H}=$ $m n$. Values for the model parameters are $\beta=0.33, \mu=$ $1, \nu=1.5, \xi=0$.

We choose a following profile function $f(r)$ in the interval $\left(r_{0}=0.01, r_{f}=100\right)$ satisfying the boundary conditions $f\left(r_{0}\right)=\pi$ and $f\left(r_{f}\right)=0$

$$
f(r)=\frac{\pi\left(r_{f}-r\right)\left(1+b_{0}\left(r-r_{0}\right)\right.}{r_{f}-r_{0}} e^{-b_{1}^{2}\left(r-r_{0}\right)},
$$

where $b_{0}, b_{1}$ are variational parameters. The profile function for the scalar field is defined in the form

$$
g(r, \theta)=\left(\frac{c_{0}}{\sqrt{a_{0}^{2}+r^{2}}}+\frac{c_{1} r}{c_{2}^{2}+r^{2}} \sin \theta\right) \frac{r_{f}-r}{r_{f}+r},
$$

where $c_{0}, a_{0}, c_{1}, c_{2}$ are variational parameters, and we keep only a leading term with angle dependence. The boundary conditions for $g(r, t)$ in the interval $\left(r_{0}, r_{f}\right)$ are fixed by the requirement of finiteness of the total energy

$$
g(0, \theta)=\text { const }, \quad g(\infty, \theta) \simeq\left(\frac{1}{r}\right)^{\alpha \geq 1} .
$$

We minimize the energy functional and find the profile functions $f(r), g(r, \theta)$. Respective energy density plots are depicted in Fig. 3. The energy values of knots are presented in Table 1, the last column $E_{\text {rat }}$.

Surprisingly, comparison of the results obtained by using energy minimization with the rational profile functions with the results obtained by using AFZ configurations implies that exact knot solutions of AFZ integrable model provide good approximate analytic solutions for our model. Notice, numeric analysis demonstrates that one has an essential qualitative difference between approximate variational solutions obtained with AFZ field configurations and with rational functions, (see Figs. 2,3). In the case of use of the rational function ansatz the energy densities for any Hopf charge are not spherically symmetric. Notice, the Lagrangian (1) has another interesting limiting case with a constrained set of the model parameters. When the parameters $\nu=\xi=0$ one has a special model without any dimensional parameters while possessing a static solution given by 8,10 , $Q_{H}=1$, with an additional constraint

$$
\frac{3}{8} \mu^{2}+\beta=0
$$

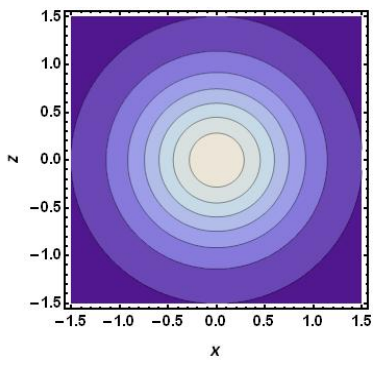

(a) $m=n=1$

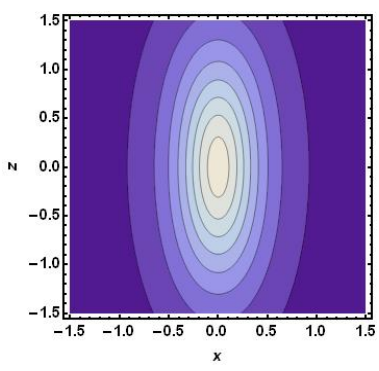

(c) $m=2, n=1$

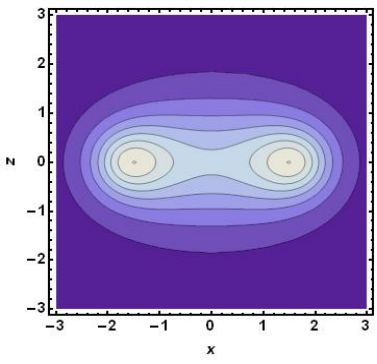

(b) $m=1, n=2$

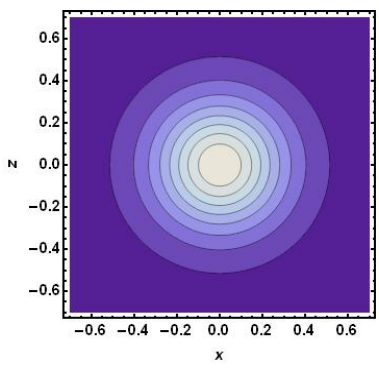

(d) $m=n=2$
FIG. 2: Energy density plots with AFZ ansatz for Hopf charge $Q_{H}=m n$

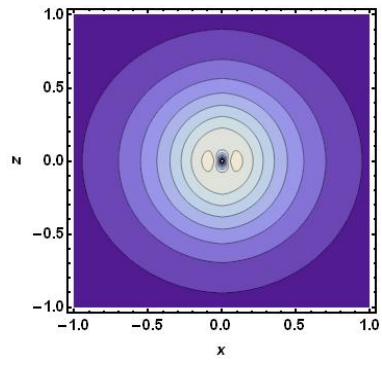

(a) $m=n=1$

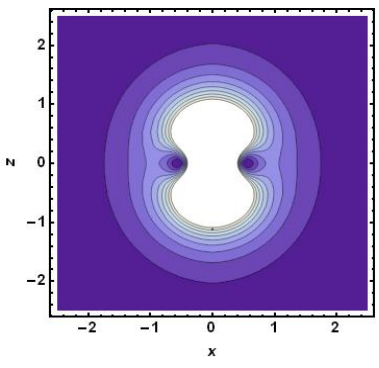

(c) $m=2, n=1$

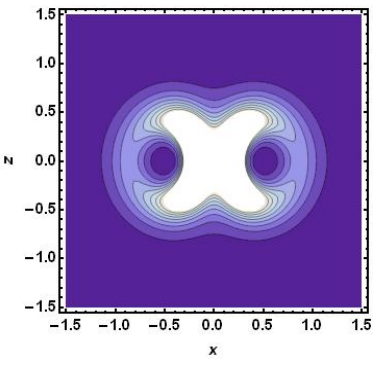

(b) $m=1, n=2$

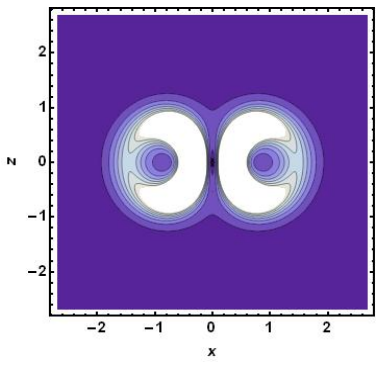

(d) $m=n=2$
FIG. 3: Energy density plots with rational functions ansatz for Hopf charge $Q_{H}=m n$

The parameter $a$ remains a free parameter. The solution has a spherically symmetric energy density

$$
\mathcal{E}_{0}=\frac{a^{2} \mu^{2}\left(r^{2}-3 a^{2}\right)}{\left(a^{2}+r^{2}\right)^{3}} .
$$

One can easily verify that a total energy vanishes identi- 
cally, so the solution represents a non-trivial zero mode. A similar solution exists in a special critical case in an extended Skyrme-Faddeev model considered in [24]. It would be interesting to study possible physical implications of such models especially in description of monopoles.

In conclusion, we have proposed a generalized SkyrmeFaddeev model with an additional scalar field. The model admits an exact knot soliton with a unit Hopf charge and inherits main features of the Aratyn-Ferreira-Zimerman integrable $C P^{1}$ model. Namely, our numeric analysis shows that AFZ knot configurations provide approximate analytic knot solutions with high symmetric properties. Notice, that the $C P^{1}$ field $\hat{n}$ realizes another non-trivial topological mappings classified by the second homotopy group $\pi_{2}\left(C P^{1}\right)=Z$, i.e., by the monopole charge. Since the model admits the Wu-Yang monopole as a singular solution, and there is an additional scalar field $\phi$ which can regularize the solution structure, it would be interesting to study possible finite energy monopole solutions. As it was suggested before, the Skyrme-Faddeev theory and its various extensions can represent low energy effective theories of QCD. Construction of a consistent extended Skyrme-Faddeev model for description of mesons and glueballs is an important issue in modern hadron spectroscopy.

\section{Acknowledgments}

The work is supported by NSFC (Grants 11035006 and 11175215), CAS (Contract No. 2011T1J31), and by UzFFR (Grant F2-FA-F116).
[1] T. H. R. Skyrme, Proc. Roy. Soc. Lond. A260, 127 (1961).

[2] I. Zahed and G. Brown, Phys. Repts. 142, 1 (1986).

[3] A. Jackson and M. Rho, Phys. Rev. Lett. 51, 751 (1983).

[4] G. Atkins, C. Nappi and E. Witten, Nucl. Phys. 228,552 (1983).

[5] N. Manton and P. Sutcliffe, Topological solitons. Cambridge Monographs on Mathematical Physics. Cambridge University Press, (2004).

[6] R. A. Battye and P. M. Sutcliffe, Rev. Math. Phys. 14, 29 (2002).

[7] D. T. J. Feist, P. H. C. Lau, N. S. Manton, Skyrmions up to Baryon Number 108, arXiv: 1210.1712 [hep-th].

[8] L. D. Faddeev and A. J. Niemi, Nature 387, 58 (1997).

[9] R. A. Battye and P. M. Sutcliffe, Phys. Rev. Lett. 81, 4798 (1998).

[10] Y. M. Cho, Phys. Rev. Lett. 87, 252001 (2001).

[11] A. Niemi, Phys. Rev. D61, 125006 (2000).

[12] Y. M. Cho, Phys.Lett. B616, 101 (2005).

[13] Y. M. Cho, B. S. Park and P. M. Zhang, Int.J.Mod.Phys. A23, 267 (2008).

[14] Y. M. Cho, H. W. Lee and D. G. Pak, Phys. Lett. B525,
347 (2002).

[15] Y. M. Cho, D. G. Pak, J. Korean Phys. Soc. 38, 151 (2001).

[16] H. Gies, Phys. Rev. D63, 125023 (2001).

[17] L. A. Ferreira, J. Jaykka, Nobuyuki Sawado, Kouichi Toda, Phys. Rev. D85 105006 (2012).

[18] L. A. Ferreira, P. Klimas, W. J. Zakrzewski, Phys. Rev. D84, 085022 (2011).

[19] L. A. Ferreira, A. C. Riserio do Bonfim, JHEP 1003:119 (2010).

[20] M. Hirayama, C.-G. Shi, Phys. Rev. D65 (2002) 065008.

[21] D. A. Nicole, J. Phys. G4, 1363 (1978).

[22] H. Aratyn, L. A. Ferreira and A. H. Zimerman, Phys. Rev. Lett. 83, 1723 (1999).

[23] A. F. Vakulenko, L. V. Kapitanski, Sov. Phys. Dokl. 24, 433 (1979).

[24] E. De Carli, L. A. Ferreira, J. Math. Phys. 46012703 (2005).

[25] R. A. Battye, M. Haberichter, Classically isospinning Hopf solitons, arXiv:1301.6803 